\title{
The BRAIN CMB polarization experiment
}

\author{
G. Polenta ${ }^{\mathrm{a}, *}$, P.A.R. Ade ${ }^{\mathrm{b}}$, J. Bartlett ${ }^{\mathrm{c}}$, E. Bréelle ${ }^{\mathrm{c}}$, L. Conversi $^{\text {a }}$, P. de Bernardis ${ }^{\mathrm{a}}$, \\ C. Dufour ${ }^{\mathrm{c}}$, M. Gervasi ${ }^{\mathrm{d}}$, M. Giard ${ }^{\mathrm{e}}$, C. Giordano ${ }^{\mathrm{a}}$, Y. Giraud-Heraud ${ }^{\mathrm{c}}$, B. Maffei ${ }^{\mathrm{b}}$, \\ S. Masi ${ }^{\text {a }}$, F. Nati ${ }^{\text {a }}$, A. Orlando ${ }^{b}$, S. Peterzen ${ }^{a, f, g}$, F. Piacentini ${ }^{a}$, M. Piat ${ }^{\text {c }}$, \\ L. Piccirillo ${ }^{\mathrm{b}}$, G. Pisano ${ }^{\mathrm{b}}$, R. Pons ${ }^{\mathrm{e}}$, C. Rosset ${ }^{\mathrm{c}}$, G. Savini ${ }^{\mathrm{b}}$, G. Sironi ${ }^{\mathrm{d}}$,
}

\author{
A. Tartari ${ }^{\text {d }}$, M. Veneziani ${ }^{\text {a,c }}$, M. Zannoni ${ }^{\text {d }}$ \\ a Dipartimento di Fisica, Universit Ã di Roma "La Sapienza", Roma, Italy \\ ${ }^{\mathrm{b}}$ Department of Physics and Astronomy, University of Cardiff, UK \\ " APC, College de France, Paris, France \\ 'Dipartimento di Fisica, Universit Ã di Milano "Bicocca", Milano, Italy

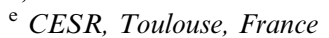 \\ ${ }^{\mathrm{f}}$ ISTARS, Pagosa Spings, CO, USA \\ g Agenzia Spaziale Italiana, Roma, Italy
}

Available online 31 January 2007

\begin{abstract}
In the standard cosmological scenario tensor perturbations of the metric produce a rotational component in the Cosmic Microwave Background polarization, called B-modes. The expected signal is of the order of $\sim 0.1 \mu \mathrm{K}$, well beyond the sensitivity of current experiments. In this paper, we introduce the BRAIN experiment, a bolometric interferometer devoted to measure the B-modes polarization of the CMB from Concordia station in Antarctica. This experiment will take advantage of both high sensitivity of bolometer arrays and low level of systematic effects of interferometers. We report the results obtained during the first campaign we carried out this austral summer at Dome-C. Using a pathfinder experiment, we demonstrated that a cryocooler based cryogenic system can work in such environmental conditions. We also carried out preliminary observations of the atmospheric emission at $145 \mathrm{GHz}$ from Dome-C, and during the next campaign we'll extend the measurements to all the Stokes parameters $I, Q, U, V$ all over the winter.
\end{abstract}

(c) 2006 Elsevier B.V. All rights reserved.

PACS: 98.80.-k; 95.75.Hi; 95.75.Kk; 98.70.Vc

Keywords: Cosmology; Polarimetry; Interferometry; Background radiation

\section{Contents}

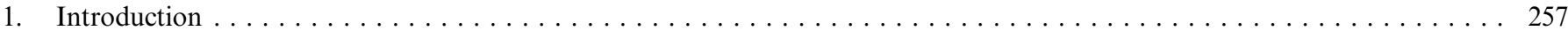

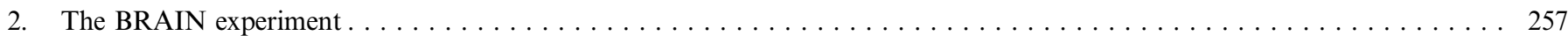

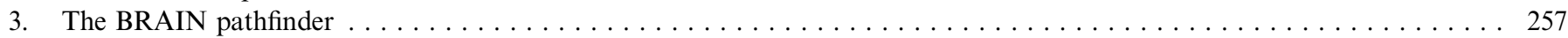

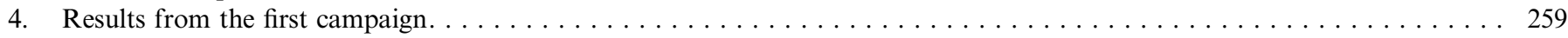

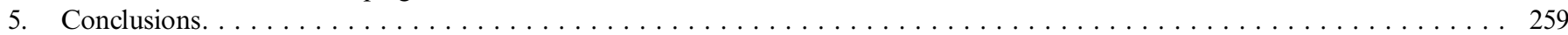

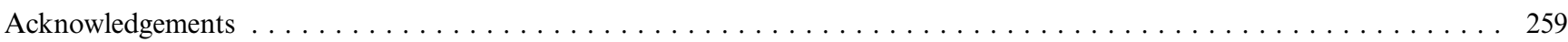

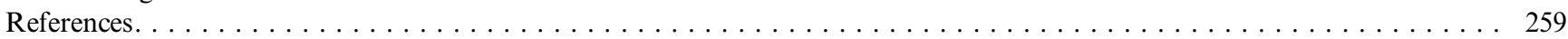

\footnotetext{
* Corresponding author.

E-mail address: gianluca.polenta@roma1.infn.it (G. Polenta).
} 


\section{Introduction}

Cosmic Microwave Background polarization offers a unique opportunity to study the physics of the early universe. Starting from unpolarized incoming radiation with local quadrupole anisotropy, Thomson scattering will produce linear polarization in the outcoming photons. In analogy with electric and magnetic fields, the polarization is divided in a curl-free component (E-modes) and a curl component (B-modes) (Kamionkowski et al., 1997; Zaldarriaga and Seljak, 1998). Scalar perturbations in the early universe can produce only E-modes polarization, while tensor perturbations will produce both E-modes and B-modes $(\mathrm{Hu}, 2002)$. In the standard cosmological scenario, the gravitational wave background produced during the inflationary stage is the source of a B-modes polarization component, its amplitude depending on the energy scale at which inflation occurs but of the order of $0.1 \mu \mathrm{K}$ (Turner and White, 1996), and its typical angular scale of the order of $2^{\circ}$. This is one of the most important observable for both Cosmology and Fundamental Physics, probing the physics at Grand Unification Theory energy scale $\left(\sim 10^{16} \mathrm{GeV}\right)$, see e.g. $(\mathrm{Hu}, 2002)$. Another source of $\mathrm{B}$-modes polarization is the gravitational lensing effect of the E-modes polarized CMB photons by large scale structures during the path from last scattering surface to us (Kaplinghat et al., 2003; Smith et al., 2004). This signal is still of the order of $0.1 \mu \mathrm{K}$, but its typical angular scale of $\sim 10^{\prime}$ will allow to distinguish between the two effects. This B-modes component is important as well, testing the theory of structures formation. E-modes polarization has already been detected by BOOMERanG-B03, CAPMAP, DASI, CBI and WMAP (Masi et al., 2006; Montroy et al., 2006; Piacentini et al., 2006; Barkats et al., 2005; Kovac et al., 2002; Leitch et al., 2005; Readhead et al., 2004; Page et al., 2006), while the small amplitude of B-modes make their detection a challenge for experimentalist.

\section{The BRAIN experiment}

BRAIN (Background RAdiation INterferometer) is a bolometric interferometer, combining both high sensitivity of bolometer arrays and robustness of interferometers (Piccirillo, 2003). This experiment is complementary to Clover and others imaging experiments: measuring B-modes with

Table 1

Some characteristics of Dome-C

\begin{tabular}{ll}
\hline Average air temperature & $-50.8{ }^{\circ} \mathrm{C}$ \\
Typical monthly average air temperature in summer & $-30{ }^{\circ} \mathrm{C}$ \\
Typical monthly average air temperature in winter & $-60{ }^{\circ} \mathrm{C}$ \\
Mean wind speed & $2.8 \mathrm{~m} / \mathrm{s} 5.4$ knots \\
Mean air pressure & $645 \mathrm{hPa}$ \\
Yearly precipitation range (snow) & $2-10 \mathrm{~cm}$ \\
Precipitable water vapor & $0.6 \mathrm{~mm}$ \\
Transparency at $225 \mathrm{GHz}$ & 0.94 \\
\hline
\end{tabular}

both classes of instruments will enhance our confidence in the measurements.

To reduce the impact of atmospheric emission (Hanany and Rosenkranz, 2003) we identified Concordia station in Dome-C as the best site for observations. It is a FrenchItalian base placed on the antarctic plateau at $\sim 1100 \mathrm{~km}$ from both Mario Zucchelli and Dumont D'Urville stations $\left(123^{\circ} 23^{\prime} 42^{\prime \prime} \mathrm{E}, 75^{\circ} 06^{\prime} 06^{\prime \prime} \mathrm{S}, \sim 3200 \mathrm{~m}\right.$ ASL), where low humidity and absence of wind create the best conditions for astrophysical observations. Interesting environmental characteristics are reported in Table 1 (Dall' Oglio and Valenziano, 1999; Storey et al., 2005).

It is quite clear that any manual operation during the winter in such environment will be pretty hard. Therefore we are building a fully automatized instrument. The most important problem is related to the cryogenic system, which has to be able to cool the detectors down to $\sim 0.3 \mathrm{~K}$ without manual operations. This can be achieved using a cryocooler, a mechanical refrigerator which avoid to use cryogenic liquids which need refilling.

The working principle of the BRAIN interferometer (Masi et al., 2005) is shown in Fig. 1. The incoming radiation is collected by two front horns separated by a distance $B$ and for each of them the two orthogonal polarization are separated by the Ortho-Mode Transducers (OMT). Orthogonal polarization coming from different antenna are combined using a first $90^{\circ}$ hybrid. Mixed signal are phase modulated by a phase shifter and combined again by a second $90^{\circ}$ hybrid. The outcoming signals are then focused and detected by four bolometers obtaining the following combinations of the Stokes parameters $I, Q, U$ and $V$ :

$$
\begin{aligned}
P_{1} & =\frac{1}{2}(I-Q \cdot \cos \phi+U \cdot \sin \phi \cdot \cos \alpha-V \cdot \sin \phi \cdot \sin \alpha) \\
P_{2} & =\frac{1}{2}(I+Q \cdot \cos \phi-U \cdot \sin \phi \cdot \cos \alpha+V \cdot \sin \phi \cdot \sin \alpha) \\
P_{3} & =\frac{1}{2}(I-Q \cdot \cos \phi+U \cdot \sin \phi \cdot \cos \alpha+V \cdot \sin \phi \cdot \sin \alpha) \\
P_{4} & =\frac{1}{2}(I+Q \cdot \cos \phi-U \cdot \sin \phi \cdot \cos \alpha-V \cdot \sin \phi \cdot \sin \alpha)
\end{aligned}
$$

where $\phi$ is the phase shift introduced by the phase shifter and $\alpha=\frac{2 \pi}{2} B \sin \theta \cos \beta$ is the phase difference of the signals entering the two horns. Of course, realistic systematics should be added, such as cross-polarization in the OMT and non-achromatic behaviour of both hybrids and phase shifter. A detailed study of all these effects is ongoing. The final instrument will have 256 horns and 512 bolometers divided in two frequency bands, 90 and $150 \mathrm{GHz}$.

\section{The BRAIN pathfinder}

In order to test the cryogenic system we built a pathfinder experiment, which is shown in Fig. 2. This is also valuable to verify the quality of the polarized microwave sky in Dome-C, especially to solve the issue of circular polarization, In fact, the Earth magnetic field acts on spin 1 oxygen 


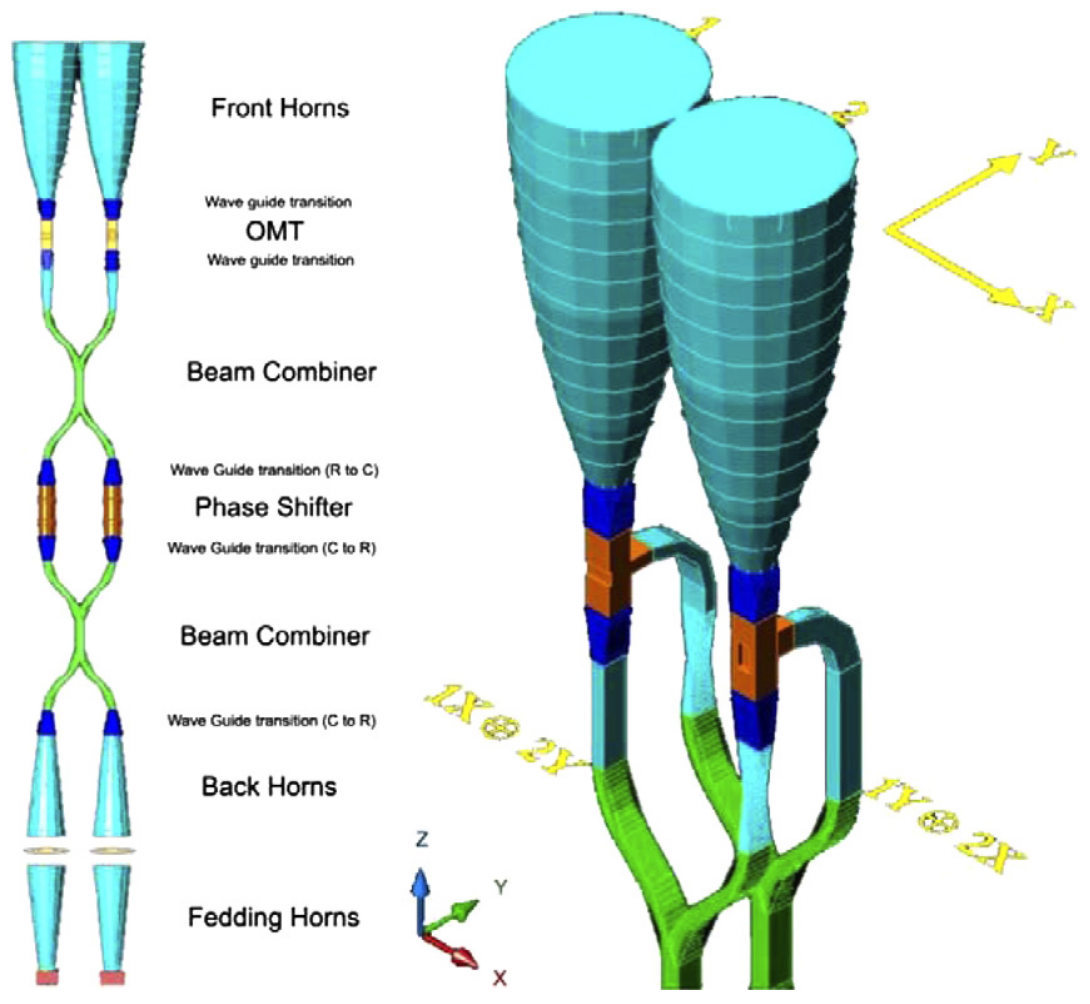

Fig. 1. Working principle of the BRAIN interferometer.
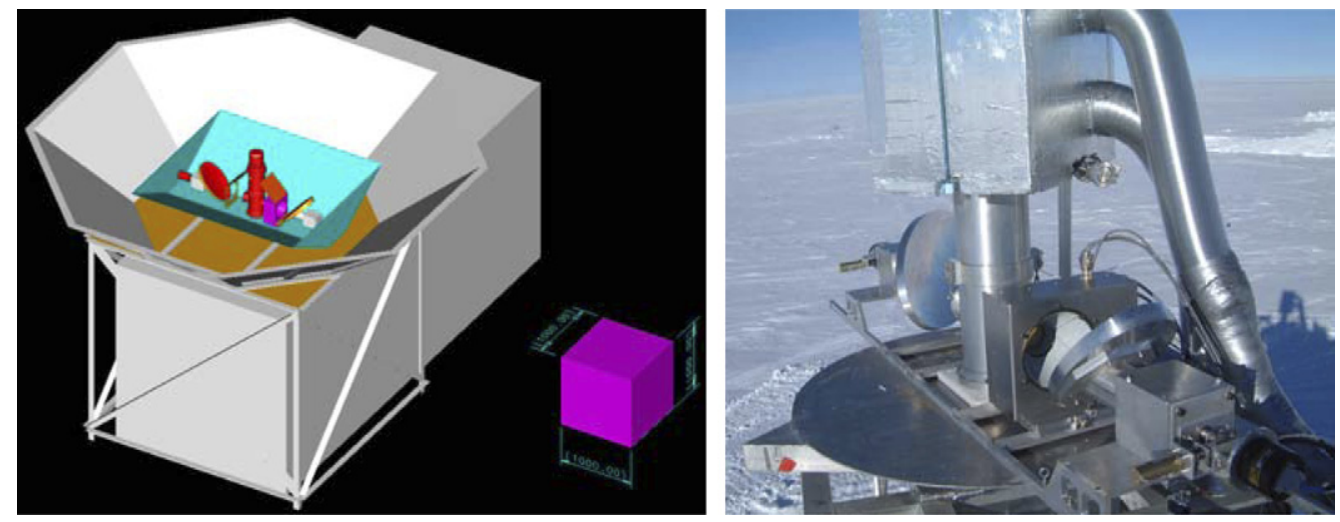

Fig. 2. Left panel: schematic of BRAIN pathfinder. Right panel: installation of BRAIN in Dome-C.

molecules producing Zeeman splitting. The relative contribution of the three emission lines (corresponding to $m=0, \pm 1$ ) depends on the angle between the direction of the magnetic field and the line of sight of the observation. The split lines corresponding to quantum number $m= \pm 1$ along the line of sight parallel to the magnetic field are fully circularly polarized. This effect has not been measured yet, however, order of magnitude calculations suggest that at $143 \mathrm{GHz}$ the amplitude of the linearly and circularly polarized components are, respectively, $10^{-9}$ and $10^{-6}$ of the total intensity (Hanany and Rosenkranz, 2003).

The instrument consists of a dry cryostat composed by a Sumitomo pulse tube mechanical cryocooler which has a limit temperature between 2 and $4 \mathrm{~K}$ depending on heat load, and a double stage ${ }^{4} \mathrm{He} /{ }^{3} \mathrm{He}$ fridge able to cool down the bolometers to $\sim 0.3 \mathrm{~K}$ (Masi et al., 2005). Two $45^{\circ}$ offaxis mirrors collect the radiation into the cryostat through two $8.5 \mathrm{~mm}$ thick UHMW polypropylene windows. One of the two channels is equipped with a polarizer and a quarter-wave plate which converts circular into linear polarization (and vice versa). It can be shown that the signal detected by the bolometer is

$S=\frac{1}{2}\left[I+Q \frac{1+\cos 4 \omega t}{2}+U \frac{\sin 4 \omega t}{2}+V \sin 2 \omega t\right]$

Hence, rotating the quarter-wave plate with frequency $\omega$, will modulate the circular polarization signal at $2 \omega$ and the linear polarization signal at $4 \omega$, allowing us to extract the four Stokes parameters and making the instrument a polarimeter sensitive to circular polarization. 

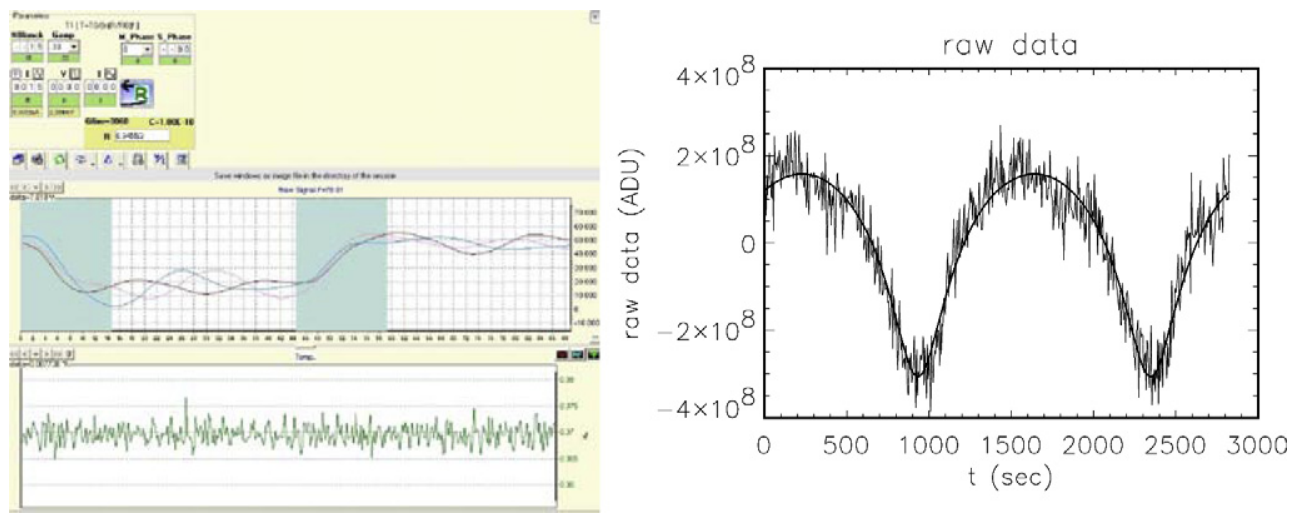

Fig. 3. Left panel: screenshot of the data acquisition software; bolometer resistance and temperature are shown be $6.34 \mathrm{M} \Omega$ and $370 \mathrm{mK}$. Right panel: raw data acquired during elevation scans and secant law prediction for atmospherical emission.

Mirrors can also move in elevation, and the whole instrument can rotate in azimuth, allowing different possibilities for both signal modulation and pointing solution. This is especially useful to extract information on the total intensity of the atmosphere as well as to observe selected regions of the sky, such as Galactic plane and Magellanic Clouds.

\section{Results from the first campaign}

We had the first BRAIN antarctic campaign in January 2006. We used most of the available time to setup the shelter to host indoor instrumentation such as compressor unit of the cryocooler, computers and data acquisition system. The shelter was placed at $\sim 300 \mathrm{~m}$ from the main station to avoid the perturbations that the worm buildings create on the lower layers of the atmosphere as well as exhausts. We installed on the roof the iridium antenna for telemetry system as well as the Wi-Fi antenna for communication with the station. The cryostat was running successfully for 3 days, and we were able to run the fridge and cool the detectors down to $370 \mathrm{mK}$ (see Fig. 3). After a few test concerning the status of all the parts of the instrument, we began observing the atmospherical emission. Unfortunately, the time available for the observation was very short and we had time only for a few elevation scans and one sky dip, made by $5^{\circ}$ step in elevation observed for $10 \mathrm{~s}$ each. The acquired data fit very well the secant law as expected for atmospherical emission (see Fig. 3), but, of course, measurements repeated at different azimuth and during different days would be needed to obtain a statistically significant estimation of the transparency of the atmosphere at $145 \mathrm{GHz}$. Nevertheless, during the day when observations were performed we obtained an upper limit for the optical depth at $145 \mathrm{GHz} \tau<0.25$ (95\% C.L.) including all the systematics. In the same spirit, by comparing the data with the calibration made in laboratory before the campaign, we can obtain a rough estimation of the incoming radiative power on the bolometers and therefore of the brightness temperature of the atmosphere as $T_{\text {atm }}=25 \pm 20 \mathrm{~K}$.

\section{Conclusions}

In this paper, we introduced the BRAIN experiment, a bolometric interferometer for B-modes of the Comic Microwave Background. Test of atmospherical emission in Dome-C and of the cryogenic system are needed and a pathfinder experiment which has been installed in Dome$\mathrm{C}$ during the past austral summer. We have preliminary indication that the quality of the site is as good as expected and the cryogenic system based on a cryocooler performed well. Next austral summer we will have our second antarctic campaign aimed to extend the measurements to all the Stokes parameters all over the winter.

\section{Acknowledgements}

BRAIN is supported by PNRA and MIUR in Italy, and by IPEV in France. This paper is dedicated to the memory of Professor Francesco Melchiorri.

\section{References}

Barkats, D. et al., 2005. ApJ 619, L127.

Dall' Oglio, G., Valenziano, L., 1999. PASA 16, 167.

Hanany, S., Rosenkranz, P., 2003. NewAR 47, 1159.

Hu, W., 2002. Phys. Rev. D 65, 023003.

Kamionkowski, M., Kosowsky, A., Stebbins, A., 1997. Phys. Rev. D 55, 7368.

Kaplinghat, M. et al., 2003. Phys. Rev. Lett. 91, 2413011.

Kovac, J.M. et al., 2002. Nature 420, 772.

Leitch, E. et al., 2005. ApJ 624, 10.

Masi, S. et al., 2006. A\&A 458, 687.

Masi, S. et al., 2005. EASPS 14, 87-92.

Montroy, T.E. et al., 2006. ApJ 647, 813.

Page, L. et al. 2006, astro-ph/0603450.

Piacentini, F. et al., 2006. ApJ 647, 833.

Piccirillo, L., 2003. Mem.S.A.It.Suppl. 2, 200.

Readhead, A.C.S. et al., 2004. Science 306, 836.

Smith, K. et al., 2004. Phys. Rev. D 70, 043002.

Storey, J. et al., 2005. EASPS 14, 7-12.

Turner, M.S., White, M., 1996. Phys. Rev. D 53, 6822.

Zaldarriaga, M., Seljak, U., 1998. Phys. Rev. D 58, 023003. 